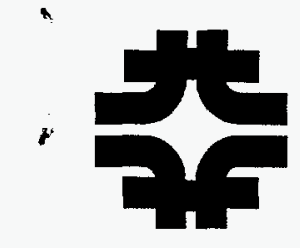

CONF-9608/2--11

Fermi National Accelerator Laboratory

FNAL/C-96/233-E

FERMILAB-Conf-96/233-E

M96050410

CDF

\title{
New Evidence for Rapidity Gaps Between Jets and Diffractive $W$ and Dijet Production at CDF
}

\author{
K. Goulianos \\ Representing the CDF Collaboration \\ Rockefeller University \\ 1230 York Avenue \\ New York 10021 \\ Fermi National Accelerator Laboratory \\ P.O. Box 500, Batavia, Illinois 60510
}

"

OCT 22 १९s

OSTI

August 1996

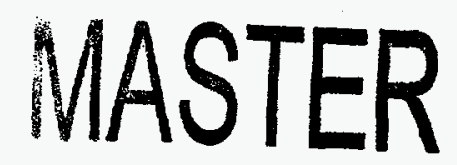

D:CTIZUTION OF RIIS DOCUMENT IS UNLIMTTEO

Presented at 1996 Annual Divisional Meeting (DPF 96) of the Division of Particles and Fields of the American Physical Society, Minneapolis, Minnesota, August 10-15, 1996. 


\section{Disclaimer}

This report was prepared as an account of work sponsored by an agency of the United States Government. Neither the United States Government nor any agency thereof, nor any of their employees, makes any warranty, express or implied, or assumes any legal liability or responsibility for the accuracy, completeness or usefulness of any information, apparatus, product or process disclosed, or represents that its use would not infringe privately owned rights. Reference herein to any specific commercial product, process or service by trade name, trademark, manufacturer or otherwise, does not necessarily constitute or imply its endorsement, recommendation or favoring by the United States Government or any agency thereof. The views and opinions of authors expressed herein do not necessarily state or reflect those of the United States Government or any agency thereof.

\section{Distribution}

Approved for public release: further dissemination unlimited. 


\section{DISCLAIMER}

Portions of this document may be illegible in electronic image products. Images are produced from the best available original document. 
FERMILAB-CONF-96/233-E

\begin{tabular}{l|l} 
RU 96/E-29 & CDF/PUB/JETS/PUBLIC/3821
\end{tabular}

\title{
NEW EVIDENCE FOR RAPIDITY GAPS BETWEEN JETS AND DIFFRACTIVE W AND DIJET PRODUCTION AT CDF
}

\author{
K. GOULIANOS \\ The Rockefeller University, 1230 York Avenue \\ New York, NY 10021, USA \\ (Representing the CDF Collaboration)
}

\begin{abstract}
We present new evidence for events with a central rapidity gap between jets, and new results on diffractive $I F$ and dijet production in $p \bar{p}$ collisions at $\sqrt{s}=1.8 \mathrm{TeV}$.
\end{abstract}

We report new evidence for dijet events with a central rapidity gap (RG) between jets and results on diffractrive (tagged by a forward RG) $W$ and dijet production in $p \bar{p}$ collisions at $\sqrt{s}=1.8 \mathrm{TeV}$. The data were collected in Tevation runs $1 \mathrm{~A}$ (1992-93) and $1 \mathrm{~B}$ (1993-95) with disabled beam-beam counters, to eliminate biasing against forward RGs, and an added forward dijet trigger selecting $E_{T}^{\text {jet }}>20 \mathrm{GeV}$ and $\left|\eta^{j e t}\right|>1.8$. The results presented were obtained from $9492(29496)$ dijets with $\eta_{1} \eta_{2}>1\left(\eta_{1} \eta_{2}<1\right)$, and 8246 (4593) $W \rightarrow$ $e \nu(\mu \nu)$ events with a central $e^{ \pm}(|\eta|<1.1)$ or $\mu^{ \pm}(|\eta|<0.6)$.

The relevant CDF detector componets are the central tracking chamber $(|\eta|<1.2)$ and the calorimeters: central $\left(|\eta|<1.1 ; \Delta \eta \times \Delta \phi=0.1 \times 15^{\circ}\right)$, plug $\left(1.1<|\eta|<2.4 ; 0.1 \times 5^{0}\right)$ and forward $\left(2.4<|\eta|<4.2 ; 0.1 \times 5^{0}\right)$.

In a given $\Delta \eta$ RGs from color singlet exchange (CSE) appear as an excess over RGs from normal fluctuations in the underlying event multiplicity. In the dijet case, rather than using Monte Carlo simulations and/or fits to evaluate the excess, we determine it by comparing the multiplicity distribution with a template obtained from another process that is not expected to have CSE RGs. For opposite side (OS) dijets, $\eta_{1} \eta_{2}<0$, we use as a template the same side (SS) dijet distribution; for SS dijets, we use $W$ events, which have a relatively small amount of CSE RGs, as determined by an independent analysis based on lepton-gap correlations. The template method has the added advantage of eliminating detector related systematic uncertainties.

In our search for CSE RGs, a "particle" is defined as a track of $P_{T}>300$ $\mathrm{MeV}$ or a tower of $E_{T}$ (corrected) $>300 \mathrm{MeV}$ or $E>1.5 \mathrm{GeV}$ (in $W$ case).

In Fig. 1 we compare the track (left) and tower (right) OS (solid) multiplicity within $|\eta|<1.0$ with the (normalized) SS distribution (dashed) for $|\eta|<1.2$. The asymmetry is defined as $A=\left(N_{O S}-N_{S S}\right) /\left(N_{O S}+N_{S S}\right)$. The excess $O S$ events in the $N_{\text {track }}=0$ and $N_{\text {tower }}=0,1,2$ bins are attributed to CSE. The spreading of the tower signal into the $\mathrm{N}=1,2$ bins is presumably

\footnotetext{
${ }^{a}$ Presented at DFF'96, Minneapolis, MN, 10-15 August 1996
} 
due to calorimeter noise or due to $\gamma 5$ coming from decays of neutral particles at $|\eta|>1$. The ratio of excess $R G$ to all events is

$$
R_{\text {track }}=[2.07 \pm 0.24(\text { stat })] \% \quad R_{\text {tower }}=[2.03 \pm 0.24(\text { stat })] \%
$$

The average $R=[2.05 \pm 0.24] \%$ is larger than the $C D F^{1}$ and $D \varnothing^{2}$ values of $R_{C D F}\left[0.85 \pm 0.12(\text { stat })_{-0.12}^{+0.24}(\right.$ syst $\left.)\right] \% \quad \& \quad R_{D 0}\left[1.07 \pm 0.10(\text { stat })_{-0.13}^{+0.25}(\right.$ syst $\left.)\right] \%$ We are currently investigating the dependence of the signal on $\left|\eta^{j e t 1}-\eta^{j e t 2}\right|$.

In the $W$ sample we look for RGs within $2.4<|\eta|<4.2$. Because of the boosted cms, and the large $W$ mass requiring valence quarks from the $p$ or $\bar{p}$ to interact with the flavor symmetric pomeron, diffraction favors the (angle $\otimes$ charge)-gap correlated topology of $\eta_{l} \times C_{l}>0$ with $\eta_{l} \times \eta_{g a p}<0$ over the anticorrelated, $\eta_{l} \times C_{l}>0$ with $\eta_{l} \times \eta_{g a p}>0$, where $C_{l}$ is the sign of the charge of the lepton. Fig. $2($ left $)$ shows the correlated and anticorrelated multiplicities and their asymmetry. From the excess in the zero bin, and assuming a hard-quark pomeron structure of the form $G(\beta) \sim \beta(1-\beta)$ in calculating the acceptance (where $\beta$ is the momentum fraction of the parton in the pomeron), we find that the fraction of diffractive to non-diffractive (ND) $W$ production is

$$
R_{W}=[2.0 \pm 1.9(\text { stat } \oplus \text { syst })] \%
$$

The standard (renormalized) pomeron flux POMPYT prediction ${ }^{3}$ for a hard 3 -flavor quark pomeron is $\sim 16 \%(1.8 \%)$.

In searching for diffractive dijets, we look for an excess zero bin multiplicity in $2.4<|\eta|<4.2$ opposite the dijets using $W$ s as a template with two entries per event, one for each $\eta$-side, to reduce diffractive $W$ RGs down to $\sim(0.2 \pm 0.2) \%$. Fig. 2(right) shows the SS dijet and $W$ distributions and their asymmetry. From the excess in the zero bin we derive the diffractive to ND ratio $R=(0.46 \pm 0.09) \%$. Correcting for acceptance (assuming a hard pomeron structure), for calorimeter noise and for a possible RG signal in the $W$ sample, and including systematic uncertainties, we obtain the (preliminary) ratio

$$
R_{J J}=[0.8 \pm 0.3(\text { stat } \oplus \text { syst })] \%
$$

The standard and renormalized pomeron flux POMPYT predictions ${ }^{3}$ for a hard gluon (quark) pomeron structure are $\sim 5 \%(2 \%)$ and $\sim 0.56 \%(0.22 \%)$.

\section{References}

1. F. Abe et al., CDF Collaboration, Phys. Rev. Lett. 74 (1995) 855.

2. S. Abachi et al., D0 Collaboration, Phys. Rev. Lett. 76 (1996) 734.

3. K. Goulianos, Phys. Letters B358 (1995) 379. 

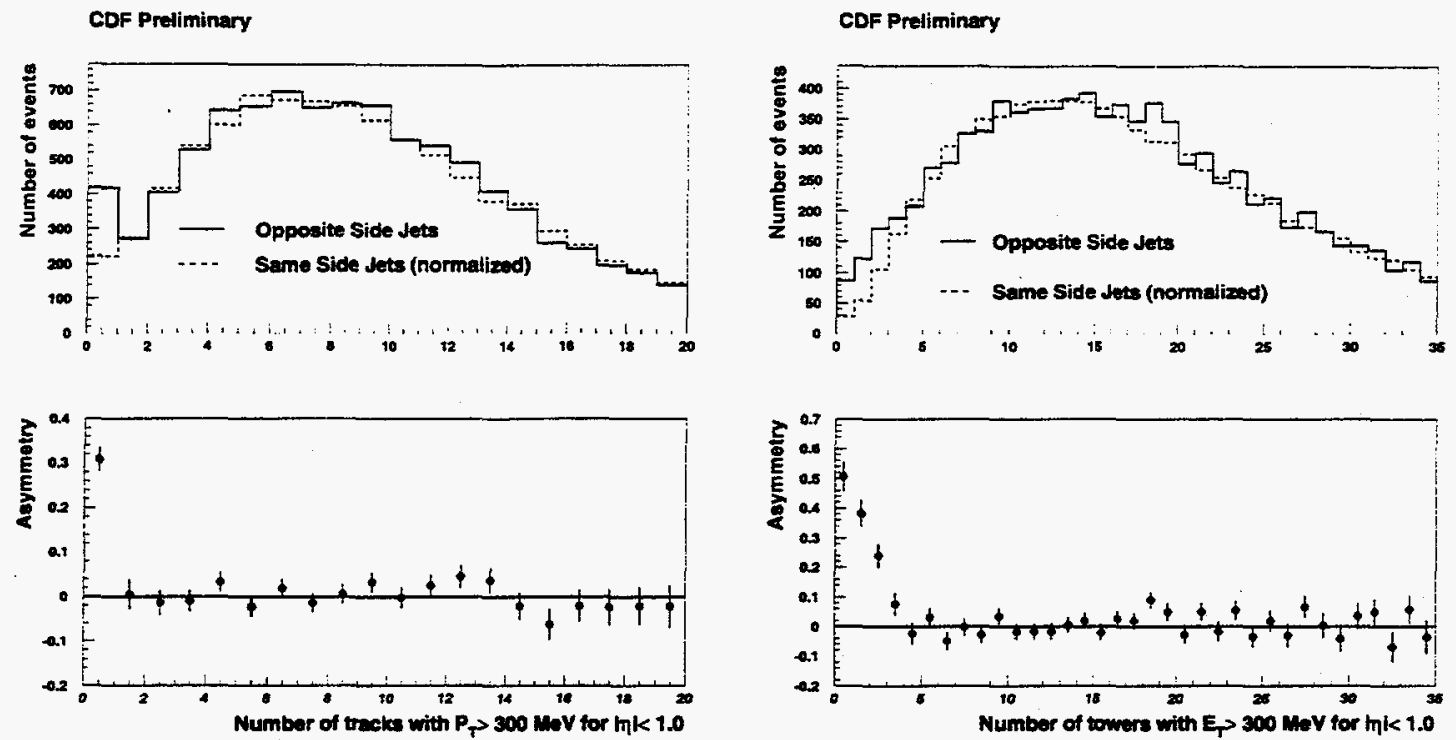

Figure 1: Dijet track (left) and tower (right) multiplicities.
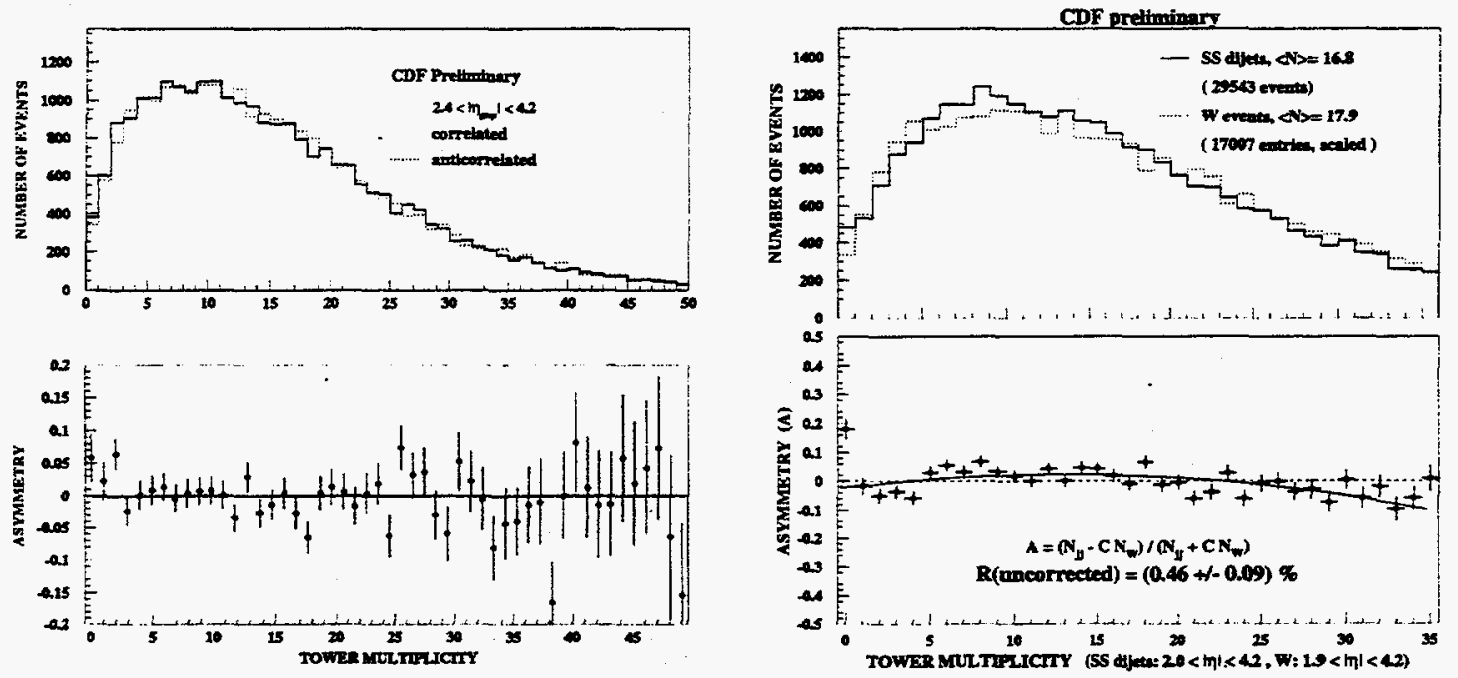

Figure 2: Multiplicity distributions within $2.4<|\eta|<4.2$ : (left) for $W$ events; (right) for SS dijet events compared to that of $W$ events. 